\title{
BioéthiqueOnline
}

\section{La prise de décision d'urgence chez les pompiers premiers répondants : une illustration de la pertinence d'une approche empirique en éthique professionnelle}

\section{Karine St-Denis}

Volume 5, 2016

URI : https://id.erudit.org/iderudit/1044291ar

DOI : https://doi.org/10.7202/1044291ar

Aller au sommaire du numéro

Éditeur(s)

BioéthiqueOnline

ISSN

1923-2799 (numérique)

Découvrir la revue

Citer cet article

St-Denis, K. (2016). La prise de décision d'urgence chez les pompiers premiers répondants : une illustration de la pertinence d'une approche empirique en éthique professionnelle. BioéthiqueOnline, 5. https://doi.org/10.7202/1044291ar
Résumé de l'article

Lors d'une urgence médicale, l'intervention se doit d'être immédiate. Le temps de délibération est court, voire inexistant. Pourtant, l'intervenant portera la responsabilité de ses décisions et de ses actions. Cette complexité de la décision d'urgence demeure peu étudiée en éthique. Pour contribuer à combler cette lacune, cet article portera sur la prise de décision chez les pompiers premiers répondants. Il présente les données issues de focus groups réalisés auprès de pompiers du Service de Sécurité Incendie de la Ville de Montréal. Dans un premier temps, cet article illustrera la compréhension de la prise de décision d'urgence de ces pompiers premiers répondants. Dans un deuxième temps, il montrera qu'une approche empirique est indispensable à l'éthicien qui s'aventure en caserne : cette approche empirique est révélatrice des confrontations éthiques des pompiers et des moyens mis en place pour neutraliser ces confrontations. 


\title{
La prise de décision d'urgence chez les pompiers premiers répondants: Une illustration de la pertinence d'une approche empirique en éthique professionnelle
}

\author{
ARTICLE (RÉVISION PAR LES PAIRS / PEER-REVIEWED) \\ Karine St-Denis ${ }^{1}$ \\ Reçu/Received: 30 Jan $2016 \quad$ Publié/Published: 23 Sept 2016 \\ Éditeurs/Editors: Vanessa Chenel \& Vincent Couture \\ Évaluateurs externes/Peer-Reviewers: Romain Pudal \& Anonymous
}

2016 K St-Denis, Creative Commons Attribution 4.0 International License

\section{Résumé}

Lors d'une urgence médicale, l'intervention se doit d'être immédiate. Le temps de délibération est court, voire inexistant. Pourtant, l'intervenant portera la responsabilité de ses décisions et de ses actions. Cette complexité de la décision d'urgence demeure peu étudiée en éthique. Pour contribuer à combler cette lacune, cet article portera sur la prise de décision chez les pompiers premiers répondants. II présente les données issues de focus groups réalisés auprès de pompiers du Service de Sécurité Incendie de la Ville de Montréal. Dans un premier temps, cet article illustrera la compréhension de la prise de décision d'urgence de ces pompiers premiers répondants. Dans un deuxième temps, il montrera qu'une approche empirique est indispensable à l'éthicien qui s'aventure en caserne : cette approche empirique est révélatrice des confrontations éthiques des pompiers et des moyens mis en place pour neutraliser ces confrontations.

\section{Mots clés}

urgence, premiers répondants, pompiers, services incendie, prise de décision, éthique professionnelle, données empiriques

\section{Abstract}

During an emergency, response has to be immediate. Deliberation time is limited, even nonexistent. But professionals are responsible for their decisions and their actions. The complexity of decision-making during an emergency is an under-researched topic in bioethics. To contribute to this topic, this article focuses on decisionmaking by firefighter first responders. It presents empirical data from focus groups with firefighters who are first responders for the Service de Sécurité Incendie de la Ville de Montréal. First, this article describes the way that firefighters understand decision-making during an emergency. Second, an empirical approach is shown to be necessary for bioethics researchers who venture to do research in fire departments: this empirical approach reveals firefighter's ethical challenges and how they can be neutralised.

\section{Keywords}

emergency, first responders, firefighters, fire service, decision-making, professional ethics, empirical data

\section{Responsabilités des évaluateurs externes}

Les évaluations des examinateurs externes sont prises en considération de façon sérieuse par les éditeurs et les auteurs dans la préparation des manuscrits pour publication. Toutefois, être nommé comme examinateur n'indique pas nécessairement l'approbation de ce manuscrit. Les éditeurs de BioéthiqueOnline assument la responsabilité entière de l'acceptation finale et la publication d'un article.

\section{Peer-reviewer responsibilities}

Reviewer evaluations are given serious consideration by the editors and authors in the preparation of manuscripts for publication. Nonetheless, being named as a reviewer does not necessarily denote approval of a manuscript; the editors of BioéthiqueOnline take full responsibility for final acceptance and publication of an article. 
Affiliations des auteurs / Author Affiliations

${ }^{1}$ Département de philosophie, Collège Lionel-Groulx, Ste-Thérèse, Canada

\section{Correspondance / Correspondence}

Karine St-Denis, karine.st-denis@sympatico.ca

\section{Remerciements}

L'auteure tient à remercier les pompiers du Service de sécurité incendie de la Ville de Montréal et la Division des premiers répondants pour leur précieuse collaboration.

\section{Conflit d'intérêts}

Aucun déclaré

\section{Acknowledgements}

The author wishes to thank the firefighters of the City of Montreal Fire Department and the First Responders Division for their valuable assistance.

\section{Conflicts of Interest}

None to declare

\section{Introduction}

Depuis décembre 2009, le Service de Sécurité Incendie de Montréal (SIM) s'est doté de pompiers premiers répondants qui desservent l'entièreté du territoire montréalais. En plus des appels incendie et de sauvetage, les pompiers de Montréal sont depuis appelés à agir comme premiers intervenants médicaux lors d'accidents de voitures, d'accidents industriels et lors d'appels d'urgence adressés à la centrale 911 par les citoyens. Ces appels vont des réactions anaphylactiques, aux accouchements d'urgence en passant par les arrêts cardio-respiratoires. Peu visible hors des appels d'urgence et des casernes, ce nouveau service de premiers répondants a transformé le travail des pompiers. Appelés antérieurement principalement pour des dommages matériels, les pompiers devenus premiers répondants doivent maintenant interagir plus directement avec les citoyens.

Outre des nouvelles connaissances cliniques, cette nouvelle interaction demande l'acquisition de compétences communicationnelles et empathiques. Cette nouvelle interaction mène aussi à la confrontation à la misère d'autrui [1]. Cet ajout d'un service de premiers répondants a donc transformé le métier de pompier, non seulement en confrontant ces professionnels à de nouveaux types d'appels d'urgence, mais aussi en les confrontant à de nouveaux questionnements, certes pratiques, mais pour certains aussi éthiques.

Le présent article portera sur la prise de décision médicale d'urgence chez les pompiers premiers répondants. II s'attardera aux éléments contextuels et humains de cette prise de décision. Notre objectif n'est pas de juger de la pertinence et de l'efficacité des protocoles cliniques élaborés par le Ministère de la Santé et des services sociaux du Québec [2] ni de juger de la qualité des interventions des pompiers. Notre objectif est plutôt de montrer comment les pompiers comprennent et vivent ces prises de décisions. Notre recherche s'inscrit donc dans le champ de l'éthique professionnelle soit d'une éthique appliquée qui s'intéresse, sans s'y limiter, aux valeurs, normes et dilemmes d'une profession [3]

Notre intérêt pour les pompiers premiers répondants provient des résultats de travaux ethnographiques antérieurs menés auprès des pompiers québécois [4-6]. Lors de ces travaux nous avons pu constater la rapidité et la diversité des prises de décision tant lors d'appels incendie que lors d'appels d'urgences médicales. Nous avons aussi pu constater la rareté des travaux consacrés aux professionnels de l'incendie [5,6] tout autant que la rareté des travaux sur la prise de décision d'urgence [7-10].

Pour contribuer à remédier à ces lacunes, nous avons entrepris une recherche exploratoire auprès de pompiers premiers répondants du SIM. Cet article présentera deux aspects de nos résultats. Premièrement, nous explorerons comment les pompiers premiers répondants comprennent la prise de décision médicale d'urgence. Nous nous intéresserons alors au temps réduit de délibération, à la prévalence des protocoles cliniques ainsi qu'aux impondérables, tant environnementaux qu'humains, qui peuvent engendrer une adaptation, voire une déviation, du protocole. Par la suite, nous 
montrerons en quoi nos données exploratoires illustrent la pertinence des données empiriques en éthique professionnelle. Nous ferons alors part de nos observations des moyens mis en œuvre pour suspendre - voire éviter - les confrontations éthiques. II s'agit là d'observations prioritaires pour l'éthicien qui souhaite s'aventurer en caserne. Dans le milieu de la sécurité incendie, nommer et analyser les confrontations éthiques peut mener à de lourdes remises en question chez l'intervenant et ainsi nuire à ses capacités d'interventions.

Avant de présenter nos résultats, attardons-nous quelques instants à la méthodologie de la recherche et à la notion de prise de décision d'urgence qui est au cœur de nos propos.

\section{Méthodologie}

Face à rareté des travaux sur les professionnels de l'incendie et sur la décision d'urgence, une recherche de nature qualitative et exploratoire fut privilégiée afin de nous : « familiariser avec les gens et leurs préoccupations »[11]. Pour ce faire, quatre équipes de pompiers ont été rencontrées lors d'entrevues de groupe [12-14]. Ces entrevues de groupe ont été réalisées dans des casernes distinctes et ont eu une durée moyenne de deux heures. Cette technique de collecte de données a été privilégiée pour deux raisons. Premièrement, elle permet de combiner entrevue et observation des normes collectives d'un groupe. Deuxièmement, cette technique a permis de respecter l'organisation de travail en équipe des pompiers.

Au total, 14 hommes et 2 femmes ont participé à ces entrevues de groupe. Nous leur avons principalement demandé comment étaient prises leurs décisions lors d'interventions d'urgence médicales et ce qui influence ces prises de décisions. Les entrevues ont été retranscrites et analysées selon une analyse de contenu thématique qui a fait émerger les catégories [25]. Les pompiers impliqués et leurs officiers ont lu et commenté l'analyse de leurs propos et leurs commentaires ont été intégrés au présent article. Ainsi, notre analyse a pu être enrichie et validée.

Finalement, il est à noter que les extraits d'entrevues présentés ici ont été édités afin d'en faciliter la lecture. Nous avons conservé des formulations qui sont linguistiquement problématiques, mais qui sont représentatives des accents et usages du langage des pompiers. Il est également à noter que cette recherche a été menée avec l'approbation d'un comité d'éthique de la recherche et du Service de sécurité incendie de la Ville de Montréal et que les principes d'éthique de la recherche, dont la confidentialité des données, ont été respectés.

\section{La prise de décision d'urgence}

Nous emprunterons ici la définition d'urgence de Sorell pour qui « An emergency is a situation, often unforeseen, in which there is a risk of great harm or loss and a need to act immediately or decisively if the loss or harm is to be averted or minimised " [16]. Cette définition est similaire à celle d'autres théoriciens de l'urgence, dont Beck [17] et Dupuy [18]. En d'autres termes, lors d'une urgence médicale, l'intervention se doit d'être immédiate: l'imminence du risque limite le temps de délibération. L'urgence exige une action rapide ; on ne peut à la fois délibérer et agir [9,19,20].

Rappelons ici que les premiers répondants ont une formation médicale limitée aux interventions d'urgence de base et que ces interventions sont régies par des protocoles cliniques. La liberté délibérative des premiers répondants est ainsi limitée. Un officier nous rappelle qu'une décision dérogeant au protocole pourrait contribuer à la détérioration de l'état de la victime puisque le niveau de formation du premier répondant ne lui permet pas d'évaluer la situation clinique dans toute sa complexité. Pourtant, l'intervenant portera la responsabilité pratique et morale de ses décisions et de ses actions. Ce contexte particulier amène Pierron à qualifier l'urgence de « cas limite de l'agir [...], elle force à questionner ce qui demeure de l'action, en ces zones de turbulences qui confondent la démesure de l'impulsivité, l'impensé du geste réflexe d'un côté, et la mesure et tempérance de l'action délibérée et réfléchie de l'autre » [20]. 
Les actions médicales réalisées par les pompiers premiers répondants correspondent bien à cette définition de l'urgence. Premièrement, leur intervention est requise lors de situations d'urgences vitales, soit des situations où le risque est imminent pour la santé et la vie du citoyen. Plus précisément, leur mandat est de procéder « à l'approche préhospitalière et à la stabilisation de l'état du patient dans l'attente des techniciens ambulanciers-paramédics » [2, p.2]. Deuxièmement, de par l'imminence du risque, l'intervention des pompiers se doit d'être rapide. Par conséquent, comme nous l'a expliqué un pompier lors de travaux antérieurs [6], le temps de délibération est réduit :

Regarde, c'est juste un exemple. On part puis on est couché. Quand on reçoit l'appel, en dedans de 5 minutes: tu reçois l'appel, tu t'habilles, tu te réveilles, tu t'habilles, t'embarques dans le camion, tu t'en vas, tu sais pas quel bâtiment, tu as l'adresse [...] Puis il te reste à peu près 2 minutes, rendu-là, en débarquant, faut que tu prennes des décisions : «C'est quoi qu'on fait? » [...] Le hamster, il tourne. Mais, il y a 3 minutes, tu dormais. Trouve un métier où est-ce qu'ils vont te demander de faire ça.

Ces propos ont été le déclencheur de la présente recherche exploratoire auprès des premiers répondants. Ces propos ont généré plusieurs questionnements éthiques notamment: Comment penser la délibération lors du temps réduit de l'urgence? Et comment parvenir à établir l'imputabilité si l'agent ne dispose pas du temps et du contexte adéquats pour mener à bien une délibération $[9,19,20]$ ? Ces questions méritaient d'être confrontées à des données empiriques afin de mieux saisir leur pertinence pour le milieu professionnel de la sécurité incendie.

\section{Présentation des résultats}

\section{La prise décision médicale d'urgence selon les pompiers}

Nos résultats exploratoires confirment que la prise de décision des pompiers se doit d'être rapide. Pour y parvenir, ils entament leurs délibérations dès la réception de l'appel qui mène à un départ immédiat de la caserne. Pour ce faire, ils se basent sur les informations reçues de la centrale 911 soit, principalement: le descriptif de l'événement, son niveau de gravité et les caractéristiques du patient. Les pompiers se fient également à leur expérience et aux types d'appels les plus communs sur leur territoire pour entamer leur délibération. Comme nous ont affirmé deux d'entre eux :

La prise de décision commence dans le camion, lors du transport [...]

C'est rare que tu arrives à un endroit puis que tu prends ta décision là.

Si tu fais ça, tu es dans le trouble.

Par contre, les pompiers sont pleinement conscients que leurs décisions et leurs planifications pourraient être modifiées à leur arrivée sur l'événement. En leurs termes : "Tu sais à quoi t'attendre, mais tu [ne] peux pas savoir ce que tu auras à faire ». Voici comment un d'entre eux nous a illustré cette possibilité d'adaptation des décisions initiales :

Des personnes inconscientes ici, les trois quarts du temps, ce sont des personnes en état d'ébriété qui s'évanouissent. On sort de la caserne puis c'est exactement le compte-rendu qu'on a : une personne de 35 ans, non-alerte à côté d'un bar, couchée dans un parking. Nous on s'en va là, comme dans $99 \%$ du temps lorsque c'est une personne intoxiquée. Mais on arrive puis c'est un arrêt cardio-respiratoire. Si la prise de décision, comme tu dis, si ta prise de décision à commence ici, là il faut que tu la "revires de bord» assez vite parce que, tout ce que tu as planifié ce n'est pas vraiment ça. C'est un couteau à deux tranchants : oui tu peux te préparer, ça donne du bon, mais, autant ça peut t'aider, autant ça peut te nuire. 
Pour les pompiers premiers répondants, ces imprévus concernant la nature de l'événement et l'état du patient distinguent leur travail de celui du personnel hospitalier et les obligent à développer une excellente capacité d'adaptation. En ce sens, Pudal affirme : «le pompier doit aussi et surtout être capable d'analyser très rapidement une situation d'intervention pour adapter sa conduite et son propos " [1]. Ce sociologue se réfère au terme grec de mètis pour qualifier cette capacité d'adaptation. Les pompiers ont l'impression que le personnel hospitalier dispose de plus amples informations, ce qui diminuerait l'exigence d'adaptation. L'un d'entre eux nous a affirmé :

C'est ça la différence que tu retrouves entre le milieu hospitalier et notre milieu : oui tu as des surprises dans le milieu hospitalier, mais quand ils reçoivent la victime normalement ils ont le background, ils n'auront pas de surprise. Ils n'auront pas une personne qui s'attendent qu'elle marche, mais non, elle est en arrêt cardio-respiratoire.

D’autres impondérables viennent modifier les délibérations entamées dès la sortie de la caserne. Tout autant que les imprévus médicaux, l'environnement physique et humain viennent transformer les décisions pratiques et, selon les pompiers, distinguent également leurs interventions de celles menées en milieu hospitalier.

Un gros point différent entre les gens du milieu hospitalier et nous, c'est qu'eux ils travaillent toujours dans leur environnement, les locaux, les outils, tout est là. [...] Nous, il faut " dealer » avec à-peu-près tout, dans toutes les conditions: dans des endroits qu'on ne connaît pas, ça peut être le bordel comme ça peut être super " clean », comme on peut être dans l'eau, comme on peut être dans neige.

Tu les vois malades dans leur environnement. Tu ne les vois pas juste malades, tu vois comment des fois c'est dégoûtant puis le stress que ça génère, sa famille, etc.

Sur certains appels, les informations sur la nature et la gravité de l'événement et l'état du patient sont simplement inconnues par les pompiers lors du départ de la caserne. Lors de ces appels, leur intervention ne pourra pas être planifiée à l'avance, elle le sera que sur place suite à la constatation de la situation. En guise d'exemple, voici ce qu'un pompier a constaté à son arrivée sur un appel logé initialement pour une fuite d'eau dans une maison de personnes âgées :

J'en ai eu un entre la toilette et le mur. Il était sur le calorifère en plein hiver, il avait des brûlures au $2^{\mathrm{e}}$ degré sur lui et il était tout " magané ॥. II n'était pas en arrêt cardiorespiratoire, mais il était juste pris là, pauvre vieux monsieur, il était pris là. Une chance qu'il avait laissé son bain coulé ; parce que l'eau coulait jusqu'en bas dans le bâtiment et c'est pour ça qu'on s'est rendu là. Sinon, il est là et personne ne le sait ; je ne sais pas quand on l'aurait trouvé.

Ces extraits nous permettent d'affirmer que la prise de décision médicale d'urgence est prioritairement comprise comme un processus qui s'entame avant l'arrivée sur les lieux ; les pompiers entament leur délibération et la planification de leur action dès le départ de la caserne. Ils pourront, notamment, discuter des protocoles applicables et des interventions similaires lors du transport vers l'événement et revoir les aide-mémoires disponibles dans leur trousse d'intervention à leur arrivée sur les lieux de l'intervention. Par contre, des impondérables environnementaux et humains et de nouvelles informations collectées sur les lieux peuvent venir modifier leurs délibérations initiales. Ils devront alors s'adapter rapidement à la situation.

\section{L'autonomie décisionnelle et l'autorité hiérarchique}

Les interventions des pompiers premiers répondants sont encadrées par les Protocoles d'intervention clinique à l'usage des premiers répondants [2]. Sous format de diagrammes, ces protocoles détaillent les actions cliniques à poser selon l'évènement médical. Nous avons demandé aux pompiers si des 
décisions pouvaient être prises hors des protocoles. Sur le plan clinique, leur réponse fut simple : "C'est rendu tellement encadré que d'après moi pour avoir à prendre une décision qui, parce que c'est hors protocole qu'est-ce que tu fais? II faut que tu en prennes une : ça doit être rare parce que c'est vraiment encadré. »

N'ayant que le statut de premiers répondants, les pompiers doivent « À l'arrivée des techniciens ambulanciers-paramédics [...] effectuer le transfert de responsabilités » [2]. Par conséquent, sur les lieux d'une intervention médicale d'urgence, les techniciens ambulanciers-paramédics et les professionnels de la santé - médecins, infirmières ou de toutes autres professions dont les compétences médicales sont supérieures - ont autorités décisionnelles sur les pompiers. Cette autorité décisionnelle est habituellement respectée et l'arrivée rapide des techniciens ambulanciersparamédics est souvent souhaitée tant pour bénéficier de leurs expertises que pour le transport rapide du patient. Mais, les pompiers s'amusent aussi de certains techniciens ambulanciersparamédics ou de certains médecins qui, à leurs yeux, font preuve d'abus d'autorité. En ce sens, certains récits d'interventions montrent que sans les pompiers, certaines décisions des techniciens ambulanciers-paramédics et des médecins ne pourraient pas se concrétiser. En ce sens, voici, le récit [21-23] fait d'une décision d'un médecin prise lors d'un accident industriel : " lls ont été obligés de couper le bras du gars. Le médecin qui est là, il a demandé au pompier de couper le bras du gars avec un ciseau. Écoute, la prise de décision c'était le médecin, mais il cherchait un volontaire pour le faire [rire]. »

L'analyse de ces extraits peut, à première vue, laisser perplexe un éthicien qui voudrait s'engager dans le milieu de la sécurité incendie. Comment parvenir à faire de l'éthique professionnelle auprès de pompiers ne valorisant pas l'autonomie décisionnelle? À quoi bon réfléchir à la responsabilité morale de l'agent si celle-ci est gouvernée par un protocole ou une décision hiérarchique? Ces questions trouvent réponse pour qui sait être à l'écoute des données empiriques. En effet, les discours des pompiers offrent des fenêtres de réflexions éthiques à ceux qui savent réellement entendre. L'éthicien doit, par contre, être patient et trouver moyen de passer outre les discours communs gardien de la cohérence de l'identité collective des pompiers.

\section{Discussion sur la pertinence d'une démarche empirique en éthique professionnelle}

Passer outre les discours communs demande doigté, prudence, patience, mais surtout confiance mutuelle. Reconnaître qu'un manquement aux protocoles n'est pas toujours synonyme d'erreur ou reconnaître que certaines interventions peuvent confronter professionnellement et subjectivement l'intervenant n'est pas chose facile. Pour l'éthicien qui s'aventure dans ces zones d'incertitudes inconfortables, la limite entre les discours communs et l'affirmation de l'identité morale individuelle est mince et difficilement tangible. Seule une démarche empirique menant à une connaissance du milieu, de son vocabulaire, de ses manières d'être et de faire, mais surtout une compréhension de ses interdits - pour ne pas dire de ses tabous - permet de saisir et d'atteindre ces zones d'incertitudes inconfortables sans ébranler les discours communs nécessaires à la cohérence professionnelle.

À titre d'exemple de l'importance d'être à l'écoute des données empiriques, voici un échange entre premiers répondants où la valeur personnelle du respect de la dignité humaine devant la mort est affirmée tout en conservant l'adhésion collective au protocole clinique dictant l'obligation de la réanimation. La fin de ce récit est forte de sens : ici dire ses choix moraux est rendu possible grâce à un changement dans les protocoles cliniques survenu en 2007.

\footnotetext{
${ }^{1}$ Nous utilisons ici la notion de récit pour montrer la force symbolique de cette narration. II est fort peu probable qu'un pompier parvienne à couper un bras avec un ciseau. Mais ce récit est porteur de sens : il dit plus que l'intervention, il dit la perception du rôle des pompiers et des médecins.
} 
On est reparti pour un arrêt cardio-respiratoire. C'est une dame de 102 ans, elle vient de décéder dans les bras de sa fille. II n'y a rien de plus beau, rien de plus naturel.

- Faut que tu la masses pour qu'elle revienne.

- $\quad$ II suffit qu'il y en ait un dans la place qui te dise : «Tu la réanimes ».

- Y faut la réanimer.

J'arrive puis une femme dit: "C'est ma mère, elle est décédée, on attend le médecin ». Mais moi, j'ai mon protocole. Encore une fois, quand on parle d'un peu de vécu : j'étais jeune pompier : "Si je ne masse pas, je vais être dans la $\mathrm{m} . .$. , pis....". Donc, mon partenaire et moi on avait déposé la dame à terre, on commençait à préparer nos manœuvres puis ça a bien tombé, le médecin d'Urgence-santé ${ }^{2}$ est arrivé [...]. Lui, comme médecin, il a pu déclarer le décès, mais c'était... J'arriverais sur cette même intervention-là avec 20 ans d'expérience je dirais qu'on la sortirait même pas du lit parce que ... Puis, les protocoles ont changé, là le vouloir verbal d'un membre significatif peut arrêter les manœuvres.

Cette adhésion collective aux normes et aux protocoles n'est pas une adhésion aveugle, irréfléchie. Les interventions habituelles des pompiers - le combat d'incendie, le sauvetage nautique, les accidents industriels, etc. - se caractérisent par leur dangerosité. Face à cette dangerosité, le respect des normes de pratiques et des normes de santé et sécurité au travail (SST) est garant tant de la sécurité et de la survie des pompiers que de la réussite des interventions.

Mais, il est possible de croire que cette adhésion collective aux normes et protocoles va au-delà de la protection physique des pompiers et de l'efficacité des interventions. Cette adhésion collective semble aussi protéger des confrontations éthiques qui pourraient devenir difficiles à supporter. À titre d'exemple, il est plus difficile pour un pompier d'être confronté à la douleur de la conjointe d'un homme en arrêt cardio-respiratoire que de mener les manœuvres de réanimation. Voici un échange entre 2 pompiers à ce sujet :

- Moi j'aime mieux être sur la mécanique, de compter puis de respirer que d'être le troisième pompier qui est pris pour expliquer à la madame l'autre bord qu'est-ce qui ce passe. J'ai travaillé avec des pompiers qui avaient un don pour être le troisième pompier : " Ok. Madame, là je vais vous expliquer... » Mais moi, personnellement, je suis plus à l'aise avec le mécanique.

- Ça des fois on est exposés à ça puis on n'a pas le choix, on est au pied du mur et ça nous tente pas là.

Dans le milieu de la sécurité incendie, la réponse doit être rapide et efficace, la réussite des interventions en dépend. Les pompiers doivent donc trouver moyen de neutraliser - ou du moins de suspendre - leurs confrontations éthiques pour maximiser la rapidité et l'efficacité de leurs interventions. Selon Desmond [22], un sociologue et pompier forestier américain, la survalorisation de la technique est un moyen d'éviter les confrontations éthiques. Il qualifie cette survalorisation d'illusion du libre arbitre, en d'autres termes, d'illusion utile qui conditionne notre réflexion. Pour illustrer ces propos, Desmond offre un compte rendu évocateur d'une journée de formation où le décès en fonction de collègues fut l'objet d'une analyse technique détaillée : " À la fin de la journée, nous avions établi une longue liste de ce que ces pompiers auraient dû faire. Nous aurions fait mieux, assurions-nous l'inspecteur et nous-mêmes. Nous aurions survécu » [22]. Ici l'illusion du libre arbitre

2 II est à noter qu'Urgence-santé n'a plus de médecins sur la route depuis 2000. 
conditionne les pompiers en les amenant à croire que l'excellence technique leur permettra d'éviter la mort.

Cette illusion du libre arbitre n'est pas spécifique aux pompiers. Elle a été analysée auprès d'autres professionnels de l'urgence, policiers et techniciens ambulanciers-paramédics [6,9,23]. De plus, cette illusion ne doit pas être comprise comme une carence réflexive, une absence d'affirmation des confrontations éthiques. Au contraire, à sa manière, cette illusion du libre arbitre nomme la présence de confrontations éthiques et dit la difficulté de leur résolution.

Pour l'éthicien qui souhaite s'aventurer dans les casernes, cette illusion du libre arbitre est à la fois une fenêtre ouverte sur les confrontations éthiques et une barrière à franchir qu'avec prudence. Cette illusion du libre arbitre est protectrice de la capacité d'action des professionnels de l'urgence. Elle leur est nécessaire pour parvenir à agir en présence de la souffrance humaine. De par leur mission même de protection des citoyens, les pompiers sont dans une position éthique contradictoire : se dévouer à la sécurité des citoyens demande empathie et de par cette même empathie, le pompier ne peut demeurer neutre face à la souffrance d'autrui. Comment dès lors affirmer les confrontations éthiques tout en conservant ses capacités d'interventions lors d'urgence médicale? L'éthicien qui s'aventurera en caserne ne pourra éviter cette question.

\section{Conclusion}

À partir de données empiriques issues de focus groups réalisés auprès de pompiers premiers répondants nous avons ici montré que la prise de décision lors d'interventions médicales d'urgence nécessite une réponse rapide et que, pour ce faire, la délibération débute dès la réception de l'appel d'urgence. Chez les pompiers premiers répondants, cette délibération sera guidée par les protocoles cliniques, l'expérience professionnelle et la connaissance des interventions habituelles du territoire. À l'arrivée sur les lieux, cette délibération initiale pourrait être rapidement modifiée lors de la présence d'impondérables humains et environnementaux.

Nos données empiriques nous ont aussi permis de montrer qu'à première vue, la valorisation de du pouvoir hiérarchique et la dévalorisation de l'autonomie décisionnelle pourraient laisser perplexe un éthicien qui s'aventure en caserne. Par contre, de par leur mission même, les pompiers sont dans une position éthique complexe : se dévouer à la sécurité des citoyens demande empathie et, de par cette empathie, les pompiers ne peuvent demeurer neutres face à la souffrance humaine. Pour conserver la rapidité et l'efficacité de leurs interventions, il leur faudra donc trouver un moyen de neutraliser - ou du moins de suspendre - ses confrontations éthiques. Sur ce point, l'éthicien qui s'aventura en caserne trouvera matière et défi: Comment dès lors affirmer les confrontations éthiques tout en conservant ses capacités d'interventions lors d'urgence médicale?

Nous sommes convaincus que sans l'analyse de données empiriques préalables, il nous aurait été impossible d'atteindre cette compréhension du milieu professionnel des pompiers et de mettre en lumière cette question inévitable pour parvenir à initier une réflexion éthique avec ces professionnels de l'urgence. De par leur nature exploratoire, nos données ne permettent pas de répondre à cette question, mais invitent à la prudence.

\section{Références}

1. Pudal R. Retour de flammes. Les pompiers, des héros fatigués? Paris : Éditions La Découverte : 2016 : 50-54.

2. Gouvernement du Québec. Protocoles d'intervention clinique à l'usage des premiers répondants. Québec : Gouvernement du Québec ; 2013.

3. Goldman AH. Professionnelle (éthique). Dans Canto-Sperber (éditeur) Dictionnaire d'éthique et de philosophie morale. Paris : Presses Universitaires de France ; 2004. 
4. St-Denis K. La professionnalisation des chefs de sécurité incendie du Québec. Phase 3. La pertinence des regroupements régionaux des services de sécurité incendie. Beloeil, Association des chefs en sécurité incendie du Québec ACSIQ, 2016, 52 pages.

5. St-Denis K. Le portrait sociologique de la profession des pompiers du Québec. Une démarche inductive et mixte inspirée de la MTE. Approches inductives, $2015 ; 2$ : 156-175.

6. St-Denis K. Portrait sociologique de la profession des pompiers du Québec. Rapport de recherche. Laval : École nationale des pompiers du Québec ; 2012. 120 p.

7. Pelaccia T., Tardif J., Triby E., Ammirati C., Bertrand C., Charlin B. Comment les médecins raisonnent-ils pour poser des diagnostics et prendre des décisions thérapeutiques? Les enjeux en médecine d'urgence. Annales françaises de médecine d'urgence, 2011;1:77-84.

8. St-Denis K. Entre reconnaissance sociale et cohérence personnelle. Management des émotions chez les pompiers du Québec. Reflets, 2013;19(2):142-161.

9. St-Denis K. La force de l'urgence. Cowansville : Éditions Yvon Blais ; 2012. 148 p.

10. Viens AM., Selgelid MJ. Emergency Ethics, Farnham: Ashgate Publishing Ltd; 2012. 596 p.

11. Deslauriers J.-P. et Kérisit M. Le devis de recherche qualitative. Dans : Poupart et al. (éditeurs) La recherche qualitative. Enjeux épistémologiques et méthodologiques. Boucherville : Gaëtan Morin Éditeur ; 1997 : 88.

12. Burgess RG. In the Field. An Introduction to Field Research. London : Routledge ;1984 (2007).

13. Olivier de Sardan J-P. La rigueur du qualitatif. Les contraintes empiriques de l'interprétation socio-anthropologique. Louvain-La-Neuve : Bruylant-Academia ; 2008.

14. Poupart J. L'entretien qualitatif : considérations épistémologiques, théoriques et méthodologiques. Dans Poupart Jean et al. (éditeurs) La recherche qualitative. Enjeux épistémologiques et méthodologiques. Boucherville : Gaëtan Morin Éditeur : 1998.

15. Paillé P. Mucchielli A. L'analyse qualitative en sciences humaines et sociales. Paris : Armand Colin ; 2012.

16. Sorell T. Morality and emergency. Proceedings of the Aristotelian Society. 2002;103:21-37.

17. Beck U. La société du risque. Sur la voie d'une autre modernité. Paris : Champs Flammarion ; 1986 (2003).

18. Dupuy J.-P. Pour un catastrophisme éclairé. Quand l'impossible devient certain. Paris, Éditions du Seuil 2004.

19. Manning PK. Violence and the police role. Annals of the American Academy of Political and Social Science, 1980;452:138-139.

20. Pierron J-P. L'urgence. Dans Guineret Hervé (éditeur) L'action. Délibérer, décider, accomplir. Paris : Ellipses Éditions Marketing S.A. ; 2006 : 114.

21. Bensa $A$. Les sciences sociales face à l'évènement. La fin de L'exotisme. Essai d'anthropologie critique. Toulouse : Anacharsis Éditions : 2006 : 171-195.

22. Desmond M. Des morts incompétents. Actes de la recherche en sciences sociales, $2006 ; 165$ (5) : 8-27.

23. Tangherlini T. Heroes and lies: storytelling tactics among paramedics. Folklore, 2000;111:4366. 